\title{
Biosensor for Label-free DNA Quantification based on Functionalized
}

\section{LPGs}

Helena M.R. Gonçalves ${ }^{a}$, Luis Moreira ${ }^{a}$, Leonor Pereira ${ }^{a, c}$, Pedro Jorge ${ }^{b}$, Carlos Gouveia $^{\mathrm{b}}$, Paula Martins-Lopes ${ }^{\mathrm{a}, \mathrm{c}}$, José R. A. Fernandes ${ }^{\mathrm{a}, \mathrm{b}}$ *

${ }^{\text {a }}$ University of Trás-os-Montes and Alto Douro, P.O. Box 1013, 5000-911 Vila Real, Portugal

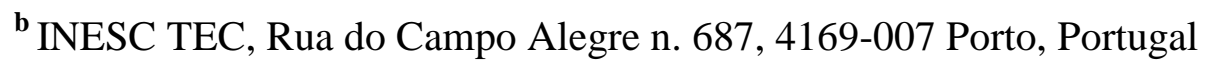

${ }^{\mathbf{c}}$ University of Lisboa, Faculty of Sciences, BioISI- Biosystems \& Integrative Sciences Institute, Campo Grande, Lisboa, Portugal

\section{Corresponding Author}

*jraf@utad.pt

\begin{abstract}
A label-free fiber optic biosensor based on a long period grating (LPG) and a basic optical interrogation scheme using off the shelf components is used for the detection of in-situ DNA hybridization. A new methodology is proposed for the determination of the spectral position of the LPG mode resonance. The experimental limit of detection obtained for the DNA was $62 \pm 2 \mathrm{nM}$ and the limit of quantification was $209 \pm 7 \mathrm{nM}$. The sample specificity was experimentally demonstrated using DNA targets with different base mismatches relatively to the probe and was found that the system has a single base mismatch selectivity.
\end{abstract}

Keywords: Biosensing, Label-free, Detection, Quantification, DNA. 


\section{Introduction}

Monitoring biological processes is not an easy task. Biological organisms are quite complex, and, as such, it is not surprising that there is still much to uncover. The discovery of the DNA molecule and its biological role can definitely be perceived as a landmark in science. Ever since the determination of the DNA molecules there has been an increasing interest in understanding the genome and in detecting differences among the DNA sequences. One of the most active research area is the specific DNA detection. Indeed these biosensors can be determinant in fields like criminology, civil defence, pharmacogenetics, pathology, genetics and food safety (Wang et al., 2006). It is easy to see the tremendous breakthrough associated with the development of a specific DNA sensor that provides an analysis almost in real-time. Let us consider disease management, as an example of DNA sensing application. The common techniques for evaluating the presence of a virus are based on culture processes (Cambau et al., 1999), that usually take several days to obtain a result, in situations where a fast answer is a pressing need (Chanteau et al., 2003). Although PCR based methodologies can be used, they require specific knowhow and special equipment in order to perform the experiments. The current available DNA biosensor applications can be classified into four broad categories: sequencing, mutation, detection, and matching detection (Palecek, 2004).

Nowadays, the biosensor technology faces some great challenges and the number of publications that illustrate an attempt to overcome the common drawbacks are growing. Indeed, there are some problems inherent to biosensing technology, namely the high cost, the low reproducibility and sensitivity and lack of reusability (Murieta and Patón, 2012). All these drawbacks are somewhat known and some strategies have been developed in order to overcome them. Wang et al. (2006) produced an optical fiber-based DNA sensor that showed good specificity and sensitivity. Pollet et al. (2009) developed a SPR-fiber optic biosensing that uses Streptavidin, PEG and biotin-labelled aptamers. This system is reusable and the DNA concentration has a linear trend with SPR wavelength shift of $0.5-5 \mu \mathrm{M}$. Lubin et al. 
(2006) successfully demonstrated an E-DNA-based sensor approach for detecting DNA authentication tags that are associated with paper or drugs. On another research field, Lai et al. (2006) achieved the specific-sequence detection of non-purified amplification products of the gyrB gene of Salmonella typhimurium. Wu et al. (2013) produced a specific DNA sensor that presents good reproducibility. Indeed even though some progresses have been made in order to overcome the drawbacks associated with biosensors it remains difficult to surpass all at once.

The DNA biosensor described in this work allows the discrimination between nonspecific and specific binding even with just a single mismacth, without the use of expensive blocking agents and fluorophores. Moreover, the strategy here developed allows to overcome some of the common drawbacks described in the biosensing technology, like reproducibility, specificity, high cost, recyclying, among others. When considering only the LPG sensing technology, this sensor is also quite competitive, since the background noise that usually makes it difficult to discriminate between a specific and a non-specific binding was significantly decreased by using ultrapure water instead the typical TE and PBS buffers.

This is a label-free, simple, specific and reproducible biosensor for DNA detection and quantification, using optical fiber long-period grating (LPG) as physical support for the DNA immobilization, recognition and hybridization.

\section{Material and Methods}

\subsection{Reagents}

All oligonucleotides used in this work were purchased from Frilabo. The stock solutions were prepared with ultrapure water and stored at $-20^{\circ} \mathrm{C}$. Each solution contained 100 $\mu \mathrm{M}$ of each oligonucleotides.

The oligonucleotides sequences are the following: 
Probe DNA: 5'-C6-Aminolink-GGTGAAATGGGCACCGAACACACGC-3'

Complementary DNA (Target 1): 5'-GCGTGTGTTCGGTGCCCATTTCACC-3'

One Base Mismatch (Target 3): 5'-GCATGTGTTCGGTGCCCATTTCACC-3'

Non-Complementary DNA (Target 2): 5'-AAAAAAAAAAAAAAACCATTTCACC-3'

One Base Mismatch (Target 6): 5'- TCG TGT GTT CGG TGC CCA TTT CAC C- 3'

Three Base Mismatch (Target 7): 5' - TTT TGT GTT CGG TGC CCA TTT CAC C - 3'

Two Base Mismatch (Target 8): 5'- GCG TGT GTT CGG TGC CCA TTT CAC T -3'

Three Base Mismatch (Target 9): 5'- GCG TGT GTT CGG TGC CCA TTT CTT T - 3'

For each experiment a suitable amount of the stock solution was diluted in saline phosphate buffer (PBS: $10 \mathrm{mM}$ sodium phosphate; $120 \mathrm{mM} \mathrm{NaCl} ; 2.7 \mathrm{mM} \mathrm{KCL}$; pH 7.4) in order to obtain the following concentrations: $0.50 ; 0.25 ; 0.125$ and $0.0625 \mu \mathrm{M}$. All other chemicals were used without further dilutions.

The cleaning solution used before each experiment was composed by Ethanol $70 \%$ $(\mathrm{v} / \mathrm{v})$ and $1 \%$ Hydrochloric acid (v/v) in a (1:1) ratio. Additionally the restringing solution was a mixture of PBS with 0.1x Saline-Sodium Citrate (SSC) e $0.1 \%$ Sodium dodecyl sulphate $(\mathrm{SDS})$ in a $(1: 1)$ ratio.

After each cycle the LPG was cleaned using a diluted solution of Nitric Acid $\left(\mathrm{HNO}_{3}\right.$ $1: 3)$.

\subsection{Preparation of the LPGs}

The gratings were fabricated in-house by using a home built electric-arc setup (Rego et al. 2001). The LPG`s were written in a standard SMF-28 fiber (Corning, USA) using an axial tension of $50.0 \mathrm{mN}$, an electric current of $9 \mathrm{~mA}$ and duration of $0.5 \mathrm{~s}$ for each electric arc. During fabrication the fiber is illuminated with a white light source and the spectrum is monitored in real time by an optical spectrum analyzer (OSA), Ando AQ-6315B, with a maximum wavelength resolution of $0.5 \mathrm{~nm}$. The sequence arc discharge/fiber displacement is 
repeated several times until the required attenuation value for the resonance peak is obtained. In order to produce a resonant wavelength at approximately $1550 \mathrm{~nm}$, corresponding to the 6th order cladding mode, the grating period was set to $398 \mathrm{~mm}$, this choice was based on previous work (Caldas et al., 2009). The sensor length is around $40 \mathrm{~mm}$ depending on the number of periods necessary to achieve the desired resonant wavelength and loss (between 80-90 periods). Due to the fabrication process, each LPG is unique and has an unique spectral shape and value of attenuation but their sensitivity to refractive index depends essentially on the cladding order mode excited which is the same for all the sensors used. A LPG is considered usable when, using the previous fabrication conditions, its resonance wavelenght is between $1535 \mathrm{~nm}$ and $1565 \mathrm{~nm}$, its loss is better than $-15 \mathrm{dBm}$ and the half height width is lower than $20 \mathrm{~nm}$. Under these boundaries it was verified that the refractive performance of the different LPGs fabricted was identical.

Indeed, the sensitivity of the LPG to the surrounding refractive index (SRI) was also studied for the range between 1.317-1.374, corresponding to the range of interest for biological measurements (Zibaii et al., 2010). The LPG was characterized in terms of SRI and its transmission spectra for different SRIs were recorded. The SRI was changed by using pure solutions of solvents with known refactive index for the wavelenght of $1550 \mathrm{~nm}$ : methanol for 1.317, pure water for 1.318 , aceton for 1.350 , ethanol for 1.352 and isopropanol for 1.374 . The data was recorded with the LPG at constant temperature of $37^{\circ} \mathrm{C}$ measured with a T-type thermocouple. A linear dependence between the wavelenght position of the resonance and the SRI was observed. A linear equation was fitted to the data using a least square based algorittm and a correlation coefficient of $r^{2}=0.9987$ was obtained. The slope value of this fitting corresponds to a measure of the LPG SRI sensitivity and was found to be $62 \pm 2 \mathrm{~nm} \cdot \mathrm{RIU}^{-1}$

The LPG sensitivity to temperature was studied using pure water as surrounding media. The temperature was changed between $36.5^{\circ} \mathrm{C}$ and $37.5^{\circ} \mathrm{C}$ in $0.1^{\circ} \mathrm{C}$ steps and, for each step, after a 20 minutes stabilization waiting period, the transmission spectra was recorded 
and analyzed. Again, a linear dependence of the resonance wavelenght position with the temperature was found. Using the same methodology that was used previously for the SRI sensitivity study, a correlation coefficient of $r^{2}=0.9919$ was obtained. The fitting slope corresponds to the LPG temperature sensitity and was found to be $0.100 \pm 0.003 \mathrm{~nm} \cdot{ }^{\circ} \mathrm{C}^{-1}$. The temperature was measured using T-type themocouple calibrated for the temperature range between $0{ }^{\circ} \mathrm{C}$ and $100^{\circ} \mathrm{C}$. These sensitivity values are the values obtained for the LPG used in the following experiments. These are tipical values that can be obtained for the fabrication conditions used and are a comprimise between the values of the two senstivities

\subsection{Hybridization and Stripping Procedure}

The LPGs were inserted in a glass chamber and the mechanical settings were set in a fixed value to avoid sensitivity losses or fluctuations. Before performing any tests it was necessary to properly clean the LPG surface in order to guarantee that no changes in the refractive index were due to the chemical interactions between the LPG surface and the chemical compounds added through the process. This cleaning procedure was accomplished by immersion in an ethanol $70 \%(\mathrm{v} / \mathrm{v})$ and hydrochloric acid $1 \%(\mathrm{v} / \mathrm{v})$ solution in a 1:1 ratio ratio.

The LPG surface is negatively charged, so is the DNA, as such, it was necessary to use a bilinker. In this work, the bilinker chosen was Poly-L-Lysine (PLL). In each cycle the following sequence was executed: water, PLL, Probe and DNA Targets, as illustrated in the scheme of Figure 1. With the exception of water and buffer added in between the addition of an analyte, all measurements were performed over a $90 \min$ period, at $37^{\circ} \mathrm{C}$. Upon the addition of Target 1 , hybridization occurs and the target can only be removed from the LPG surface by a process called stripping. This procedure was performed at $60^{\circ} \mathrm{C}$ and included the addition of a stripping solution ( $0.1 \mathrm{x}$ SDC and $0.1 \%$ SDS in a (1:1) ratio), ethanol and water. 
When the cycle was completed it was necessary to add a diluted solution of $\mathrm{HNO}_{3}$ $(1: 3, \mathrm{v} / \mathrm{v})$ for $15 \mathrm{~min}$, to assure that all chemicals were removed from the LPG surface.

\subsection{Instrumentation}

One of the most critical technical aspect of the sensor is the necessity to provide a uniform liquid based surrounding media through the optical fiber lateral surface in the LPG zone. These conditions were provided using a closed flow cell chamber (Queirós et al., 2014). The reaction chamber was made of a glass tube with $5 \mathrm{~mm}$ of outer diameter and wall thickness of $1 \mathrm{~mm}$. In both ends of the tube a Teflon ${ }^{\circledR}$ rod with $25 \mathrm{~mm}$ diameter was inserted providing mechanical stability, passage to the fibre and liquid input and output by manual injection. The fiber was maintained at constant strain using suitable fiber clamps. With such chamber, the accounted wavelength variations can only be due to the interaction of the solution with the LPG surface. The chamber capacity is of $750 \mu \mathrm{L}$ and the same volume of each solution was injected into the chamber in order to maintain the sample volume constant at all stages. The optical data acquisition was performed using a fibre optic interrogation unit manufactured by Fibersensing®, model BraggMeter FS2200SA as shown in Figure 2, with two channels modified to allow the measuring of the transmission spectra in the spectral region between 1500 and $1600 \mathrm{~nm}$ and an optical resolution of $2.5 \mathrm{pm}$ in the high resolution mode.

The sensor apparatus was maintained at constant temperature by inserting it in a muffle (Termarks, model B 8023). The sensor temperature was measured by a T-type thermocouple positioned in contact with the sensor chamber and the temperature value was recorded by a temperature logger (Keithley ${ }^{\circledR} 740$ ) with the resolution of $0.1^{\circ} \mathrm{C}$ that was controlled remotely using a Labview ${ }^{\circledR}$ program running in the same computer used to control the BraggMeter. As a consequence, both spectra and temperature data were time stamped using the same computer so they can be easily correlated. Upon the acquisition the spectral 
results were numerically processed by a new algorithm that determined the resonance wavelength position for each acquired spectra. For each point, the temperature was measured and the wavelength was corrected according to the temperature calibration previously obtained reflecting the wavelength value at $37^{\circ} \mathrm{C}$.

\subsection{Data Processing}

In order to establish the wavelength position of the optical resonance peak in the

measured LPG optical transmission spectra a robust numeric data processing scheme is

required. The peak shape of this resonance dip is rather flat and a simple data minimum seek

introduces major errors in the results originated by the noise in the measured data. To

minimize these errors several curve interpolation methods were tested to determine the peak position, like fitting a parabola or fitting a Gaussian and the subsequent finding of the

naximum/minimum for these functions. However, the resonance spectra does not have a

symmetric shape and cannot be exactly described by these functions. As a consequence the

etrieved resonance wavelength position depends on the data set used, which is also affected

by the measurement noise. As consequence these methods fail to provide a substantial

improvement in the results.

An alternative method was used based in a technique developed for the analysis of

highly deformed reflection spectra of Fiber Bragg Gratting (FBG) sensors used by (Xavier et

al, 2015). This method was modified to fit the LPG transmission spectral features. This

pectral peak tracking algorithm is based on the determination of the geometric mean (GM) of

the spectral resonance in different levels of the resonance power curve. Once the resonance

does not have a symmetric shape, at different power levels, the calculation of the GM results

in different values from the resonance's bottom to peak. However, such variation of the GM

can be well fitted by a $3^{\text {rd }}$ order polynomial function. To enable the fitting, the spectral resonance is layered in 27 different power levels, equally spaced between $0.1 \times P_{\min }$ and 
$0.99 \times P_{\min }$. The number of power levels was chosen as a compromise between number of points necessary for a successful fit and the time necessary to process each spectra. For each power level, a geometric mean is calculated using the following equation:

$$
\lambda_{G M}^{n-l e v e l}=\sqrt[m]{\prod_{m} \lambda_{i}}
$$

The selection of the wavelengths used for the calculation of the geometric mean for each power level are done according to the following condition: $\lambda_{\mathrm{i}}$ is chosen if $P\left(\lambda_{\mathrm{i}}\right) \leq P^{\mathrm{n}-\text { level }}$. After the different geometric mean are determined, a polynomial function of $3^{\text {rd }}$ order is fitted to the data and the coefficient of zero degree is considered as the resonance wavelength position. With this methodology the effect of the resonance shape and of the measurement noise is minimized and no additional errors are introduced.

\subsection{Detection limit and quantification limit procedure}

The values of the analytical limits and system sensitivity were obtained using the following procedure. Initially some tests were performed in order to determine the concentration range where the analytical signals were statistically different. All values obtained for each concentration tested were repeated more than three times in order to assure that the signals were actually different from one another. Once this concentration range was found the linear regression method was applied to the results establishing a calibration curve. The analytical limits were then calculated using the linear trend values, namely the standard deviation $(\sigma)$ of the regression line divided by the slope $(\sigma / \mathrm{m})$. For estimation of the detection limit this value is then multiplied by a factor of three $(\mathrm{DL}=3 \times(\sigma / \mathrm{m}))$. For estimation of the quantification limit this number was multiplied by ten $(\mathrm{QL}=10 \times(\sigma / \mathrm{m}))$. The sensitivity $(\mathrm{S})$ of he analytical system was obtained through the linear regression slope.

\section{Results and Discussion}




\subsection{Temperature Dependence of the LPGs}

The development of an effective biosensor system that can be used in biological matrixes is quite difficult, as such, in order to obtain the best possible biosensor performance it is necessary to understand all the constraints imposed by the sensing components.

Even though the experiments were performed in a temperature controlled muffle, there were some minor temperature variations. In this sense and, despite the fact that Dobb et al.(2006) did not find a significant variation of the LPG with temperature, it was necessary to evaluate the temperature sensitivity of the LPGs. From the intrinsic features of the manufacturing process used, each LPG had a slightly different temperature sensitivity. From the data analysis obtained it became clear that all data collected throughout the experiments, must be corrected, to compensate for the minor changes around the set temperature of $37.0^{\circ} \mathrm{C}$, using the information obtained from the temperature calibration previously described. In each protocol step, the LPG transmission spectra is recorded during at least 30 minutes with a 2 second interval. After data processing, the first 5 minutes are excluded. This time corresponds to the interval needed for each sample to establish thermal balance with the measuring system. The remaining data points have their value corrected according to their measured temperature. Finally, this data set is used to perform an arithmetic average and the resulting value is used as the measured resonance peak position for the protocol step.

\subsection{Recovery of the LPG}

One of the major drawbacks of using this type of system is the reproducibility of the LPG, resulting from the variability's associated to the electric arc fabrication process. Indeed it is possible to see from the temperature data, that the sensor performance is affected, depending on the intrinsic properties of the resulting LPG device. Although, very similar 
LPGs can be obtained by following the selection criteria described before, the need for a very accurate measurement requires a unique calibration for each individual sensor. In this sense it would be highly desirable to devise a procedure which allows the recovery of the LPG after each sensing cycle, thereby enabling the extension of its useful lifetime. After each cycle of hybridization/stripping the LPG has immobilized on its surface the PLL and the ssDNA added in the beginning. As such, in order to start another cycle, with the guarantee that the LPG is back to its original state, it was necessary to develop a methodology that cleaned its surface without chemically altering it. Two different approaches were tested. Initially a commercial hypochlorite solution was injected. The first addition of hypochlorite was left inside the chamber for 5 minutes and, as it is possible to see from the analysis of Figure 3 a, the first addition was followed by a wavelength decrease (from i to ii). This behaviour is consistent with the deposition of some material on the LPG surface. Since only hypochlorite was added, the deposited material had to be previously present inside the chamber. The only thing that could account for this behaviour was the water repellent added in the beginning, in order to prevent a competitive mechanism between the LPG silica surface and the glass chamber. This first result for hypochlorite is consistent with the release of the repellent from the chamber and its deposition on the LPG surface. As such, the water repellent was removed from the experimental procedure. Additionally, it was also possible to observe that the second addition of hypochlorite was consistent with the removal of the deposited material from the fiber surface. These results clearly demonstrated that hypochlorite is quite effective in the removal of organic material from the silica surface.

Alternatively, the LPG was cleaned by adding a diluted solution of $\mathrm{HNO}_{3}(1: 3 ; \mathrm{v} / \mathrm{v})$. This method is quite common in laboratory practice to clean the glass material from reagents. This alternative method is a good alternative to hypochlorite, since its purity and relative concentration is easier to assess. The analysis of Figure 3b, clearly shows that this method is also quite efficient in the removal of all deposited materials from the fibre surface. Upon the 
addition of $\mathrm{HNO}_{3}$ and its removal from the chamber, it was necessary to add water. It is commonly known the exothermic nature of the dissolution of nitric acid in water and this fact can be observed by the wavelength increase upon the addition of water after the acid removal.

This recovery method was used consecutively for months in more than 20 successive cycles in a single LPG. Moreover, the obtained results clearly showed that this method proved to be effective and therefore it was the one selected for the recovery of the fibre after each assay.

\subsection{Analytical Performance of the Sensing System}

The LPG analytical performance towards DNA was tested for several weeks and, each experiment was performed at least three times, in order to assure the reproducibility of the method.

The refractive index of each substance is an intrinsic property due to its composition. Moreover it is highly dependent on the temperature. In this sensing system the measurement of the resonance wavelength was registered, once it is correlated with the refractive index of the fiber surface at each time. This way if there is a change in the refractive index, at a constant temperature, it must be due to a chemical interaction between the biological material on its surface. In order to assure that the difference observed is in fact due a chemical reaction and not just a simple mass deposition effect, upon the addition of each analyte, the system was washed and the obtained signals were subtracted to the analyte signals.

These cycles were performed continually in order to evaluate the reproducibility of the system. In Figure 4 it is possible to see an example of the typical behaviour of the sensing system for three independent cycles. Indeed, the data clearly showed that the addition of each analyte was followed by a chemical reaction that allowed the formation of "layers" in the LPG surface. Interestingly, a 44\% negative deviation between the probe signal (Probe) and 
the negative control - Target 2 (T2b) was found. Indeed this is quite interesting since the procedure applied should yield no difference between these two signals. This event can be explained by electrostatic interactions with the solution at the surface. Indeed even though there is no complementarities between the bases which prevents hybridization, both probe and target are charged and therefore electrostatic interactions are possible. A simple way to prove it was washing the system and analysing the signal afterwards. When this was performed, the probe and Target 2 (T2a) signals overlapped.

Moreover, from the analysis of Figure 4 it is also possible to ascertain the specificity of the sensing system. Indeed only when the appropriate target is placed in contact with the probe, hybridization occurs and this is reflected in an increase of the wavelength shift of $33 \%$ regarding to the wavelength shift measured for the probe. An example of this is the addition of Target 3 that did not hybridize (signal difference of $2 \%$ between Probe and T3a). The only difference between Target 3 and Target 1 is a single mismatch in the middle of the DNA chain. As such, this sensing system is so accurate that it can even discriminate the full base complementarity from one base-mismatch. Indeed, even though this is not the first sensing system that is so specific (Reisberg et al., 2006), this is a cost effective, simple system that does not require reagents addition for the DNA detection of a single base-mismatch. Moreover, the results obtained are quite reproducible, with standard deviations of $0.3 ; 0.3$; 1.2; 0.8 and $0.4 \%$ for PLL, Probe, Target 1, Target 2 and Target 3, respectively.

To further confirm the specificity of the sensing system an additional set of experiments were performed with different set of targets. Figure 5 shows the results obtained for targets with a single base mismatch at the 5'end (Target 6 - T6) and at the 3 ' end (Target 8 - T8) and for three bases mismatch at the 5' end (Target $7-\mathrm{T} 7$ ) and at the 3' end (Target 9 -T9) that can be compared directly with the results obtained for Target 1 (T1) and Target 2 (T2). The results obtained further confirm the up to single base specificity of the present biosensor. 
Additionally, the sensing system can potentially have a short response time around 5 min, provided some improvements are made in the signal to noise ratrio and the signal

processing scheme. Indeed, even though the probe and targets are left to interact between 3090 minutes, it was found that the reaction takes places in the first minutes and the 5 minute response time is only limited by the number of data required to process the information.

The results so far have showed that the sensing system is able to detect with high specificity ssDNA, but it was also an objective of this work, to quantify the DNA present in a given sample. In order to do this, the experimental procedure described in Section 2.6 was followed using several concentrations of Target 1 and that allowed the calculation of the detection and quantification limit of this sensing system: Detection Limit (DL): $62 \pm 2 \mathrm{nM}$;

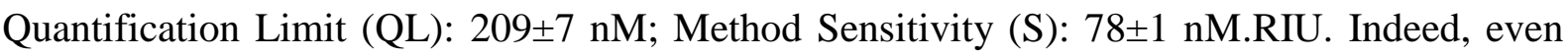
though they are lower values than previously reported (Fan et al., 2011; Komarova et al., Reisberg et al., 2005; 2006; Wu et al., 2010; Zhang et al., 2012) it is noteworthy the fact that this method allows to quantify the DNA in a sample. In order to test the applicability of the quantification procedure, a sample of Target 1 with a known concentration of $0.25 \mu \mathrm{M}$ was inserted into the chamber. According to the methodology previously described the sample was quantified in $0.24 \pm 0.01 \mu \mathrm{M}$, which is within the range of the known concentration.

\section{Conclusions}

One of the common disadvantages of using LPGs for DNA detection is the reproducibility of the results. Indeed, different LPGs have slightly different characteristics that will ultimately cause some trouble in the reproducibility of the results. In order to overcome this problem a strategy was designed, allowing the use of the same LPG in all measurements in over 20 consecutive cycles. The sensing system described is quite specific since it can discriminate between full base complementary and a single base-mismatch. 
Moreover this method provides a new, fast and simple way to not only detect DNA but also quantify in the range of hundreds of nanomolar.

Usually the quantification of DNA is based on rather expensive and time-consuming

methods. With this method it is potentially possible to have a fast, reagent free detection and

quantification of the DNA present in a given sample without the need of probe labelling or the

use of PCR. With such a method a diversity of critical applications from point of care

diagnostic, food safety and certification and security can be enabled as long there is a suitable

DNA extraction protocol.

\section{Acknowledgements}

and This work is financed by the FCT - Fundação para a Ciência e a Tecnologia (Portuguese Foundation for Science and Technology) within project UID/EEA/50014/2013 and project Biosensor Development for Wine Traceability in the Douro Region - WineBioCode PTDC/AGR-ALI/117341/2010-FCOMP-01-0124-FEDER-019439 and a PhD grant (SFRH/BD/44781/2008) 


\section{References}

Caldas, P., Jorge, P.A.S., Araújo, F.M., Ferreira, L.A., Rego, G., Santos, J.L. 2009, Meas. Sci. Technol., 20(7), 2738-2743

Cambau, E., Wichlacz, C., Truffot-Pernot, C., Jarlier V. 1999. J. Clin. Microbiol. 37, 20132015.

Chanteau, S., Rahalison, L., Ralafiarisoa, L., Foulon, J., Ratsifasoamanana, L. Carneil, E., Nato, F. 2003. Lancet 361, 211-216.

Dobb, H., Kalli, K., Webb, D.J. 2006. Optical Communications 260(1), 184-191.

Fan, H., Xu, Y., Chang, Z., Xing, R., Wang, Q.J., He, P.G., Fang, Y.Z., 2011. Biosensors and Bioelectronics 26, 2655-2659.

Komarova, E., Aldissi, M., Bogomolova, A. 2005. Biosensors and Bioelectronics 21, 182189.

Lai, R.Y., Lagally, E.T., Lee, S.H., Soh, H.T., Plaxco, K.W., Heeger, A.J. 2006. PNAS 103(11), 4017-4021.

Lubin, A.A., Lai, R.Y., Baker, B.R., Heeger, A.J., Plaxco, K.W. 2006. Analytical Chemistry 78, 5671-5677.

Murieta, I.S., Patón, A.R. 2012. BioSystems 109, 91-104.

Palecek, E. 2004. Trends in Biotechnology 22(2), 55-58.

Pollet, J., Delporta, F., Janssena, K.P.F., Jansb, K., Maesc, G., Pfeiffer, H., Weversd, M., Lammertyna, J. 2009. Biosensors and Bioelectronics 25, 864-869.

Queirós, R.B., Gouveia, C., Fernandes, J.R.A., Jorge, P.A.S. 2014, Biosensors and Bioelectronics. 62, 227-233.

Reisberg, S., Piro, B., Noel, V., Pham, M.C. 2006. Bioelectrochemistry 69, 72-79.

Rego, G.; Okhotnikov, O.; Dianov, E.; Sulimov, V. 2001. Journal of Lightwave Technology, 19(10), 1574-1579. 
Wang, X., Cooper, K.L., Wang, A., Xu, J., Wang, Z. 2006. Applied Physics Letters 89, 163901-163905.

Wu, A.H., Sun, J.J., Zheng, R.J., Yang, H.H., Chen, G.N. 2010. Talanta 81, 934-940.

Wu, N.Y, Gao, W., He, X.L., Chang, Z., Xu, M.T. 2013. Biosensors and Bioelectronics 39. $210-214$.

Xavier, J., Fernandes, J.R.A., Frazão, O., Morais, J.J.L. 2015. Composite Structures, 121

\section{Pages 83-89}

Zhang, W., Yang, T., Jiao, K. 2012. Biosensors and Bioelectronics 31, 182-189.

Zibaii, M.I., Kazemi, A., Latifi, H., Azar, M.K., Hosseini, S.M., Ghezelaiagh, M.H. 2010.

J. Photochem. Photobiol. B: Biol. 101 (3), 313-320. 


\section{Figure Captions}

Fig. 1 - Schematic representation of the protocol followed in each cycle.

Fig. 2 - Schematic of the experimental setup. The setup is constituted by a FS2200SA Braggmeter (working in transmission mode), a fluidic system containing a reaction chamber with capacity of $750 \mu \mathrm{L}$, that operate in continuous mode. The fiber was fixed with two clamps in both sides and outside the fluidic chamber. The sensing region (containing the LPG device) is place inside the fluidic chamber and maintained at a constant temperature of $37^{\circ} \mathrm{C}$. A laptop with LabVIEW software that receives and processes the optical spectra from the Braggmeter and from the temperature scanner (not represented).

Fig. 3 - Representation of the (a) hypochlorite cleaning, where i)-v) represent: water signal before the cycle, pause between additions, water after stripping, hypochlorite addition and water after hypochlorite, respectively, and (b) $\mathrm{HNO}_{3}$ cleaning where i)-vii) represent: water signal before the cycle, pause between additions, water after stripping, nitric acid addition, water after hypochlorite, signal increase due to exothermic reaction of the nitric acid and water, respectively.

Fig. 4 - Representation of the wavelength shift for the addition of each analyte to the LPG over three consecutive cycles, for PLL, Probe, Target 1 (T1), Target 2 electrostatic deposition (T2b), Target 2 signal (T2a), Target 3 electrostatic deposition (T3b) and Target 3 signal (T3a). 
Fig. 5 - Representation of the wavelength shift generated over three consecutive cycles, for PLL, Probe, Target 1, Target 2, Target 6, Target 7, Target 8 and Target 9. 


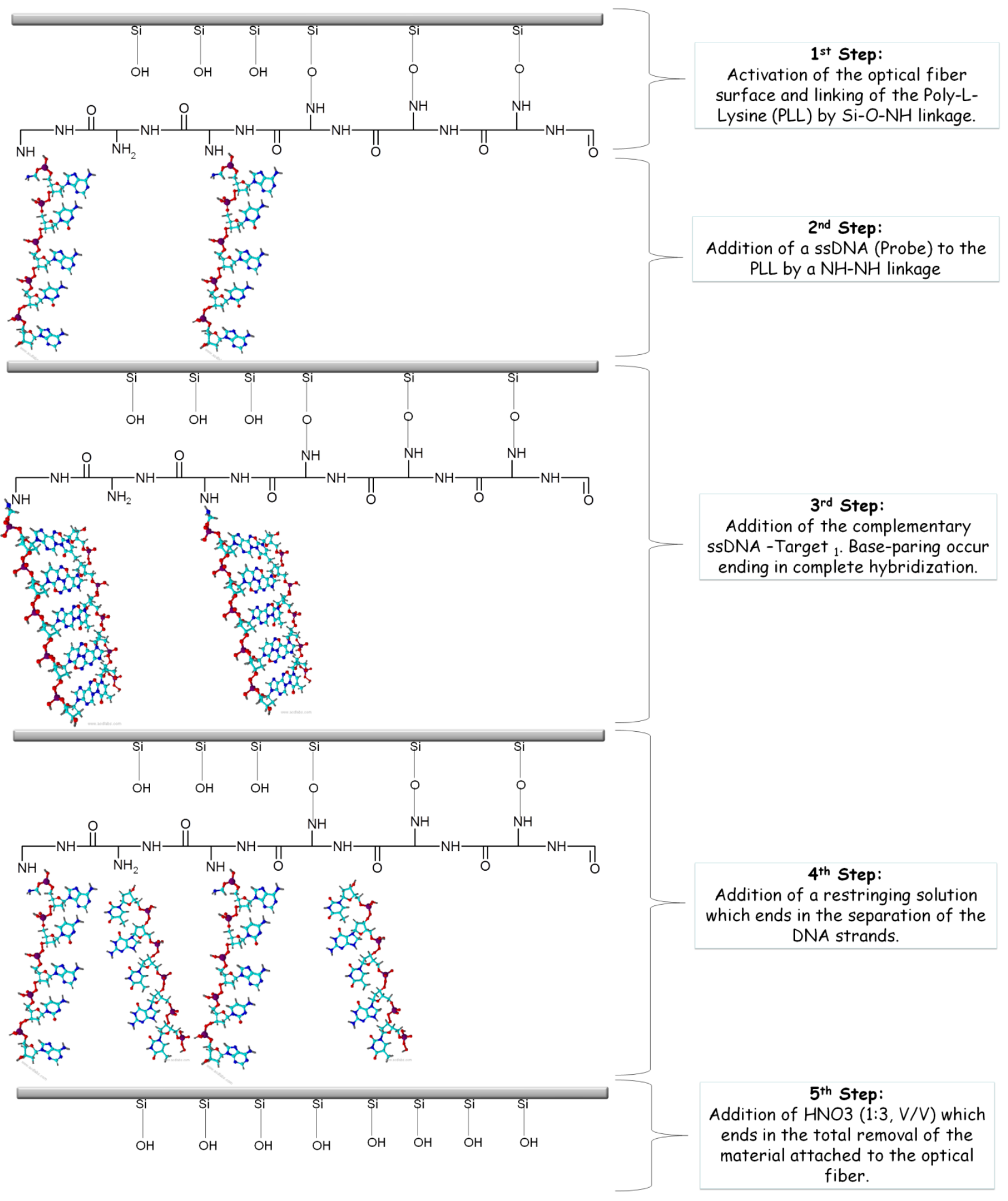

Fig. 1 - Schematic representation of the protocol followed in each cycle. 


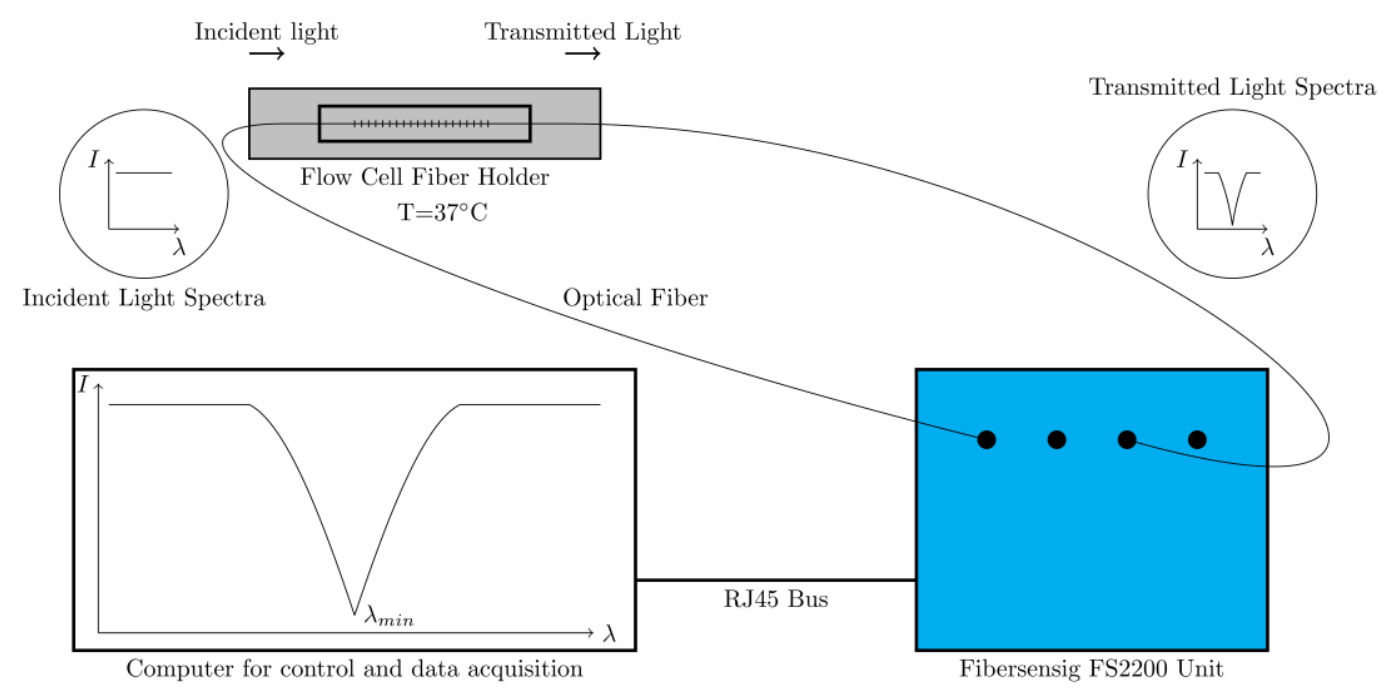

Fig. 2 - Schematic of the experimental setup. The setup is constituted by a FS2200SA Braggmeter (working in transmission mode), a fluidic system containing a reaction chamber with capacity of $750 \mu \mathrm{L}$, that operate in continuous mode. The fiber was fixed with two clamps in both sides and outside the fluidic chamber. The sensing region (containing the LPG device) is place inside the fluidic chamber and maintained at a constant temperature of $37^{\circ} \mathrm{C}$. A laptop with LabVIEW software that receives and processes the optical spectra from the Braggmeter and from the temperature scanner (not represented). 


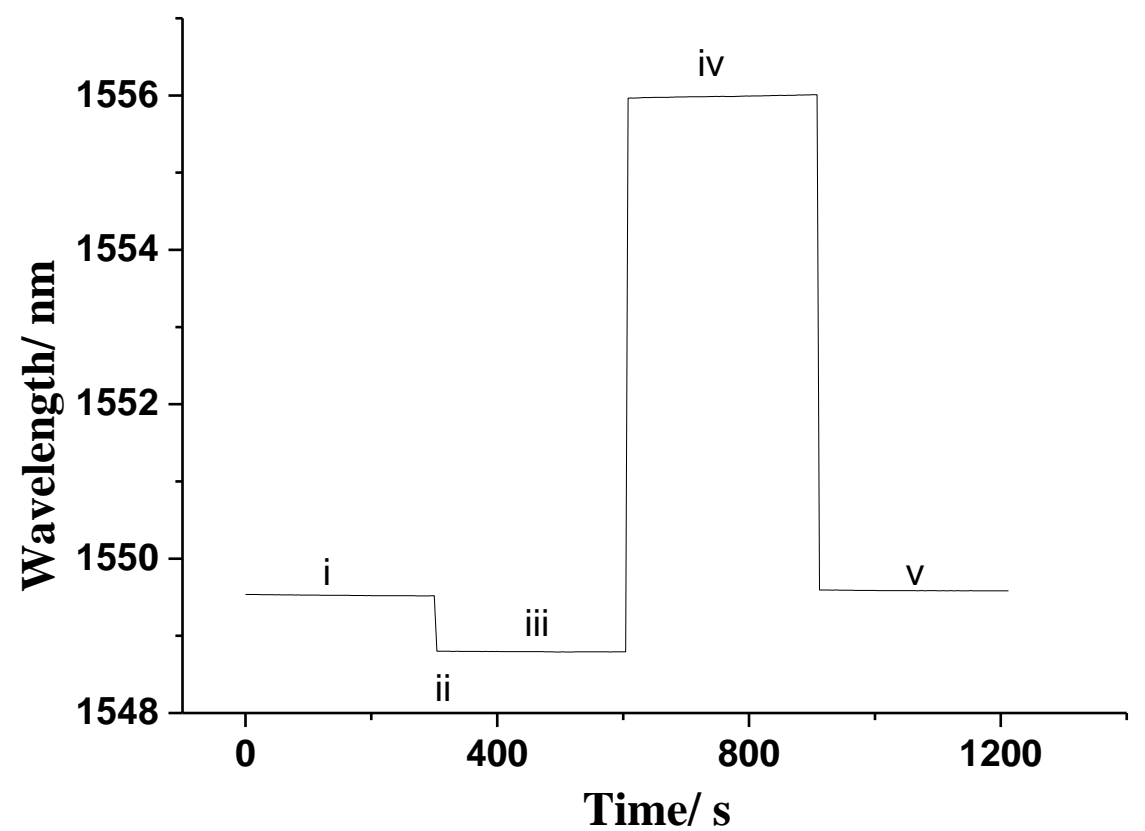

a. 


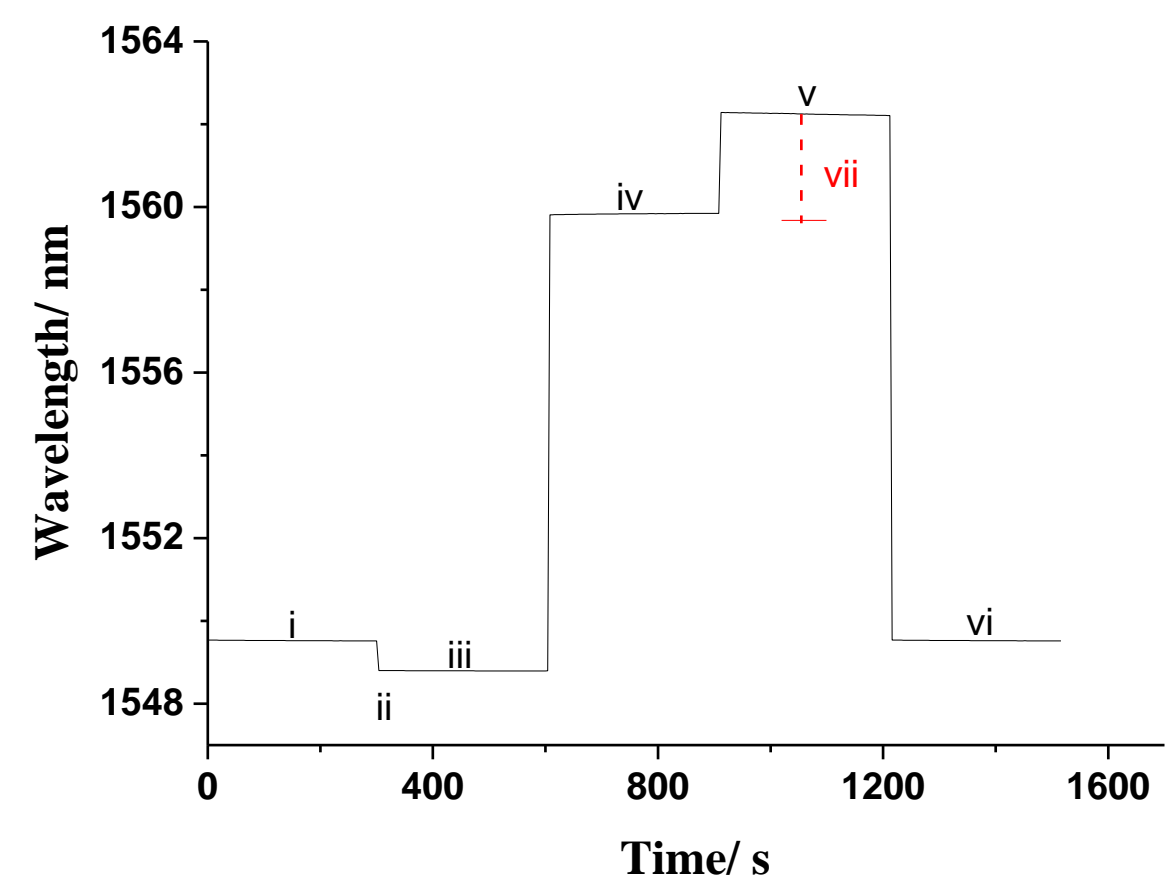

b.

Fig. 3 - Representation of the (a) hypochlorite cleaning, where i)-v) represent: water signal before the cycle, pause between additions, water after stripping, hypochlorite addition and water after hypochlorite, respectively, and (b) $\mathrm{HNO}_{3}$ cleaning where i)-vii) represent: water signal before the cycle, pause between additions, water after stripping, nitric acid addition, water after hypochlorite, signal increase due to exothermic reaction of the nitric acid and water, respectively. 


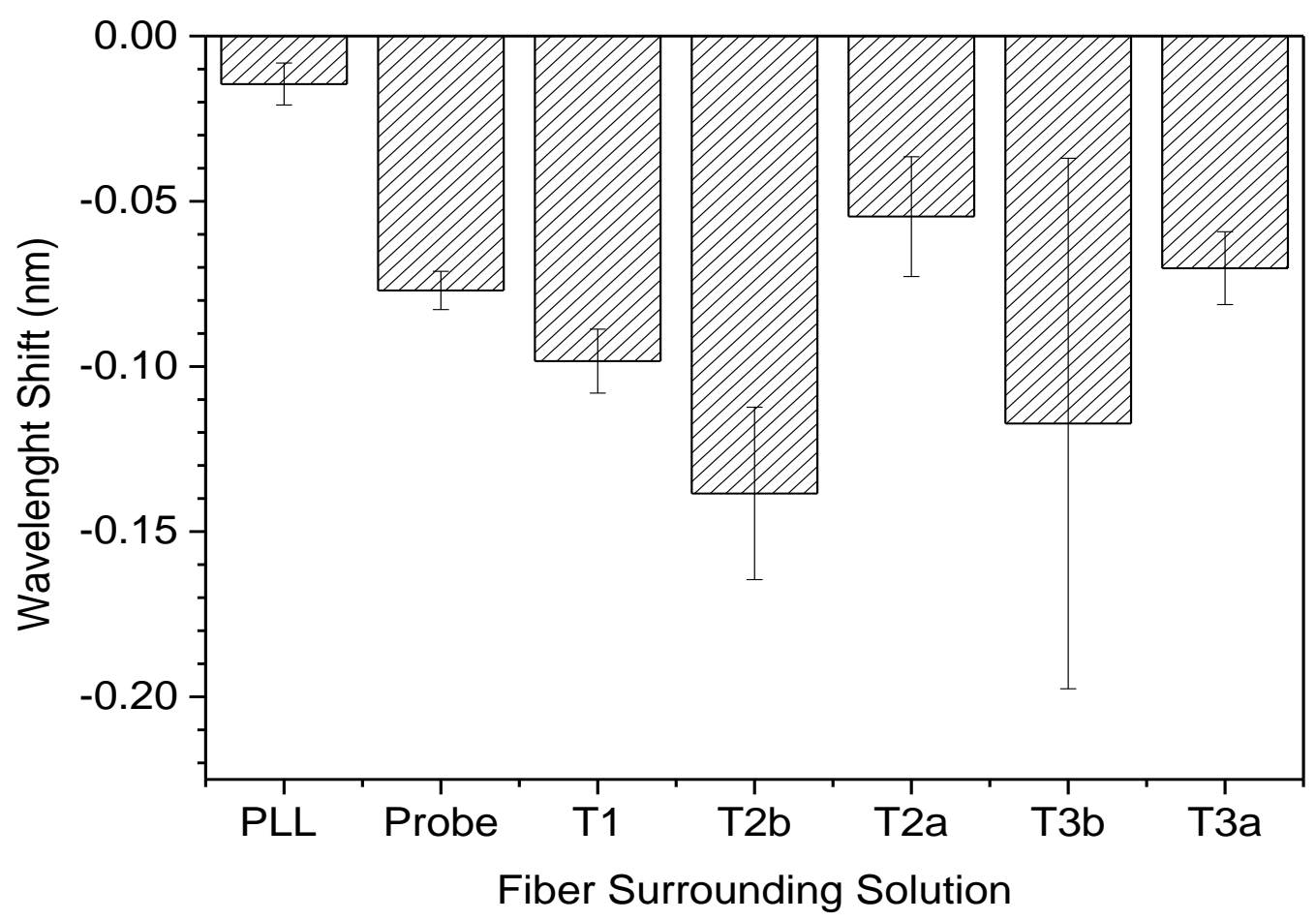

Fig. 4 - Representation of the wavelength shift for the addition of each analyte to the LPG over three consecutive cycles, for PLL, Probe, Target 1 (T1), Target 2 electrostatic deposition (T2b), Target 2 signal (T2a), Target 3 electrostatic deposition (T3b) and Target 3 signal (T3a). 


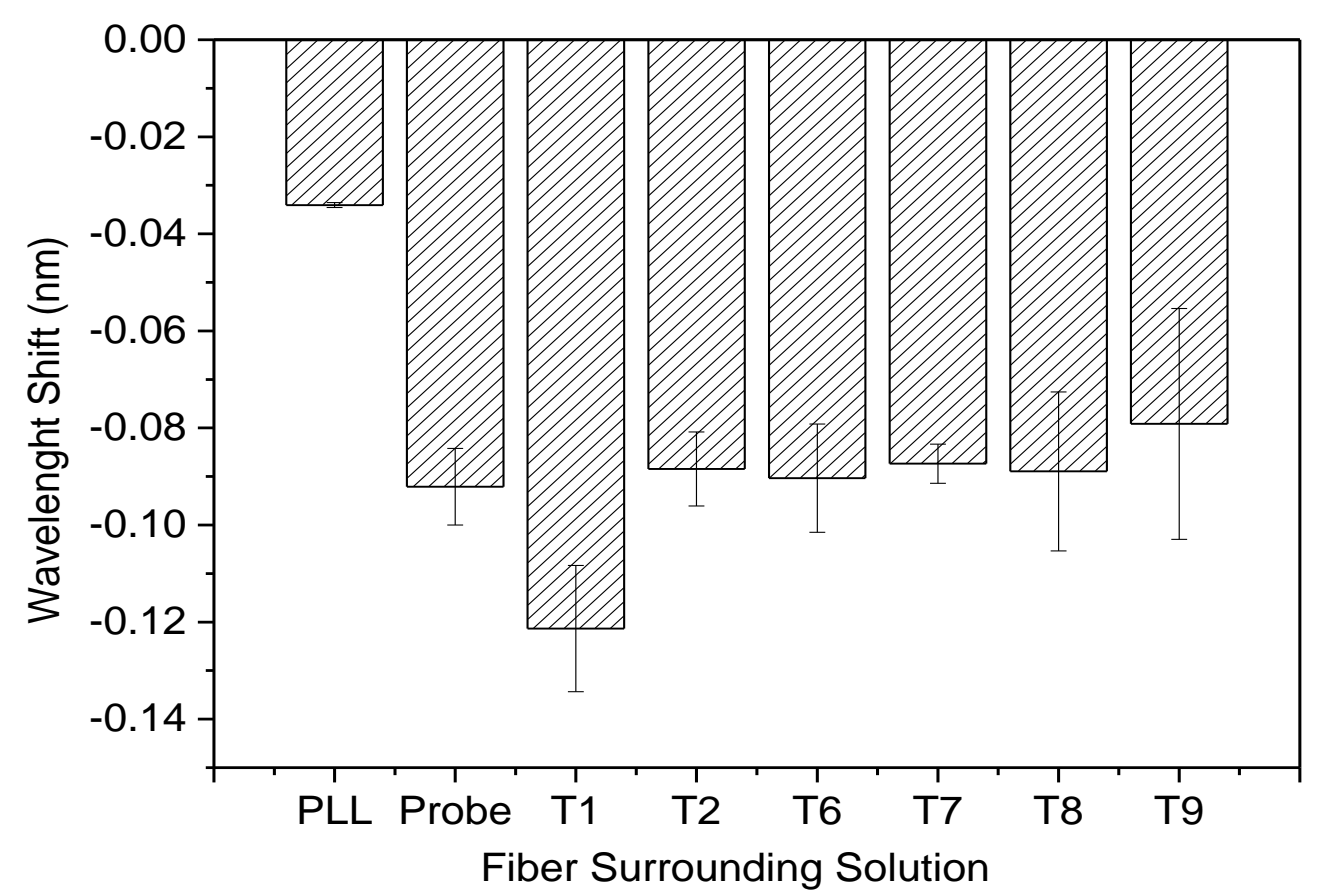

Fig. 5 - Representation of the wavelength shift generated over three consecutive cycles, for PLL, Probe, Target 1, Target 2, Target 6, Target 7, Target 8 and Target 9. 